\title{
Electrocardiographic Comparison of Dobutamine and BRUCE Cardiac Stress Testing With High Resolution Mapping in Experimental Models
}

\author{
Brian Zenger ${ }^{1,2,3,4}$, Wilson W Good ${ }^{1,2,3}$, Jake Bergquist ${ }^{1,2,3}$ Jess D Tate $^{1,2,3}$, Vikas Sharma $^{4}$, \\ Rob S MacLeod ${ }^{1,2,3}$ \\ ${ }^{1}$ Scientific Computing and Imaging Institute, University of Utah, SLC, UT, USA \\ ${ }^{2}$ Nora Eccles Cardiovascular Research and Training Institute, University of Utah, SLC, UT, USA \\ ${ }^{3}$ Department of Biomedical Engineering, University of Utah, SLC, UT, USA \\ ${ }^{4}$ School of Medicine, University of Utah, SLC, UT, USA
}

\begin{abstract}
Clinical tests to detect acute myocardial ischemia induce transient cardiac stress by means of exercise or pharmaceutical stimulation and measure electrical changes of the heart on the body surface via an electrocardiogram (ECG). Such tests assume that both stress mechanisms induce identical —or at least similar-forms of ischemia. To improve electrocardiographic detection of myocardial ischemia, we must study how varied stressing agents (pharmacological or paced stressors) change electrocardiographic signatures. We simultaneously measured electrical recordings within the myocardium, on the epicardial surface, and on the body surface. We then induced acute, controlled ischemia and monitored the electrical response. To create the hemodynamic substrate for ischemia, we applied a hydraulic occlusion to the left anterior descending coronary artery. We varied the ischemic stress with two clinical protocols, the BRUCE and dobutamine stress tests. Our results suggest significant differences in the recorded electrical signal between stress mechanisms. Differences include the location, volume, and temporal development of ischemia throughout a stress episode. These results, and the experimental means used to obtain them, are a significant breakthrough in the field with simultaneous, high density electrical recordings within the myocardium and on the heart and torso surfaces.
\end{abstract}

\section{Introduction}

Ischemic heart disease is one of the most common heart pathologies, effecting over 8 million people globally. [1] Myocardial ischemia occurs when the demand for nutrients and perfusion by the heart outweighs the available supply. This imbalance creates a supply-demand mismatch that can lead to devastating long term consequences including increased risk for myocardial infarction, cardiac arrhythmia, and sudden cardiac death.[1] For decades the electrocardiogram (ECG) has been the primary acute detection method for myocardial ischemia. [2] However, current electrocardiographic methods used to detect myocardial ischemia are mediocre at best, with reported sensitivity and specificity ranging from $50-72 \%$ and $69-90 \%$, respectively. [3] This poor performance indicates that many patients are released from clinical care unaware of their potentially life-threatening condition while others receive care they do not need. Improvements in the electrical detection of myocardial ischemia must be made to ensure patients and physicians can be confident in diagnosing and treating myocardial ischemia early to prevent potentially fatal long term consequences.

One possible source of this poor ECG-based performance may originate from different cardiac stressing mechanisms. Clinical tests induce transient cardiac stress by means of exercise or pharmaceutical stimulation and measure electrical changes of the heart on the body surface via an ECG. Such tests assume that both mechanisms induce identical, or at least similar, forms of ischemia. However, no definitive experiments have been reported that assess the electrical effects produced during different stressing mechanisms. This lack of definitive study substantiates a controlled examination of these different stressing methods.

To date, no experimental model has provided accurate and comprehensive sampling of electrical signals from controlled ischemia within the myocardial tissue, on the heart surface, and on the body surface. A model with all of these components is necessary to understand how the ischemic regions develop within the heart, and how they manifest on the body surface. For this study, our goal was to test the differences in myocardial ischemia development under two clinical cardiac stressing mechanisms, pacing the heart according to the BRUCE protocol and continuous dobutamine infusion. 


\section{Methods}

\subsection{Animal Model}

Swine and canine animal models were selected for this experimental preparation because of their similar cardiac anatomy, electrical system, and vascular structure to humans. The animals of each species were $25-35 \mathrm{~kg}$ in weight and 8 months to several years of age. The animals were purpose bred for the use in experimental research and all studies were approved by the Institutional Animal Care and Use Committee at the University of Utah and conformed to the Guide for Care and Use of Laboratory Animals. After 12 hours of fasting, the animals were sedated using an intravenous propofol bolus of 5$8 \mathrm{mg} / \mathrm{kg}$ in canines or a mixture of Telazol $(4.4 \mathrm{mg} / \mathrm{kg})$, Ketamine $(2.2 \mathrm{mg} / \mathrm{kg})$, and Xylazine $(2.2 \mathrm{mg} / \mathrm{kg})$ in swine and then intubated. Once intubated, isoflurane gas (1-5\%) was used for anesthesia. At the end of the experiment animals were euthanized while under general anesthesia, with intravenous Beuthanasia $1 \mathrm{ml} / 10 \mathrm{~kg}$. The heart was then removed for further evaluation.

\subsection{Surgical Procedure}

Following sedation, a sternotomy was performed to expose the thoracic cavity. The pericardium was opened and the heart was suspended in a pericardial cradle. Following exposure, a portion of the left anterior descending coronary artery (LAD) was dissected and a calibrated hydraulic occluder (Access Technologies, Skokie, IL, USA) was placed around the dissected portion. An atrial pacing clip was then placed on the appendage of the right atrium. Following placement of the electrical recording equipment (described below), the pericardium was sutured closed and the sternum was wired and sutured together. To limit air within the volume conductor, chest tubes were tunneled into the mediastinal, pleural, and pericardial cavities and held under constant vacuum suction. The outer layers of dermis were sutured closed and checked for potential separations. Standard laboratory markers were measured and recorded throughout the experiment including blood $\mathrm{pH}, \mathrm{PaCO}_{2}$, oxygen saturation, temperature, and blood pressure.

\subsection{Electrical Recording Equipment}

\subsubsection{Electrode Arrays}

Electrical recording equipment was all custom build at the Nora Eccles Treadwell Cardiovascular Research and Training Institute (CVRTI). The electrical signals within the myocardium were measured using transmural plunge needle arrays with 10 electrodes spaced 1.6 or $1.0 \mathrm{~mm}$ apart for left and right ventricular needles, respectively.
For these experiments, 12-25 needles were placed in the assumed perfusion bed of the LAD and concentrated on the anterior surface of the heart. The epicardial potentials were measured using a 247 -electrode sock array with evenly spaced electrodes stitched into a nylon stocking material. The distance between sock electrodes was approximately $10 \mathrm{~mm}$. The torso surface electrodes were in linear strips of 12 electrodes evenly spaced at $3 \mathrm{~cm}$ apart. Each electrode had an $11 \mathrm{~mm}$ diameter $\mathrm{Ag}-\mathrm{AgCl}$ sensor embedded in an epoxy housing with a $2 \mathrm{~mm}$ deep gel cavity. The number of strips applied to the torso surface varied between 6-10 (72-120 total electrodes) depending on the body surface area accessible for each animal.

\subsubsection{Data Acquisition}

The potentials from the sock, needle, and torso surface electrodes were recorded using a custom acquisition system. This system could record simultaneously from 1024 channels at $1 \mathrm{kHz}$ sampling rate and 12 bit resolution. Briefly, the acquisition system consisted of multiplexers, interface circuitry, and a personal computer (PC) hosting a custom program written in Labview (National Instruments, Austin, TX, USA) that managed the hardware and allowed continuous signal acquisition. A bandpass filter with cutoff frequencies at 0.03 and $500 \mathrm{~Hz}$ avoided both DC potentials and aliasing. Wilson's central terminal leads were used as the remote reference for all the unipolar signals recorded from the sock, needles, and torso surface electrodes. Prior to each experiment, calibration signals were recorded for each channel.

\subsection{Ischemia Intervention Protocols}

During each experiment, several transient ischemic interventions could be induced. Each of these interventions lasted between 8-15 minutes and were followed by a 30minute rest period. The BRUCE exercise stress was simulated by increasing paced heart rate a set amount above resting heart rate every three minutes for fifteen minutes. This increase in heart rate was predetermined from average increased heart rates during BRUCE stress protocols reported in the literature. [4] The occlusion percentage was fixed throughout the stress interval. The intervention was terminated with the presence of a sequence of three or more premature ventricular contractions. During the dobutamine stress protocol, the animal was continuously infused at a a sequence of doses, each for three minutes. Dosages followed the standard clinical dobutamine stress testing protocols [5] and the intervention again lasted 15 minutes or until a sequence of three premature ventricular contractions occurred. 


\subsubsection{Image Acquisition and Segmentation}

After each experiment, the intact torso was imaged with a clinical 3-Tesla MRI (Seimens Medical) for gross anatomy and electrode positions. Following the full torso scan, the heart was excised and scanned with a 7-Tesla MRI scanner (Bruker BIOSPEC 70/30, Billerica, MA) using FISP (Fast Imaging with Steady-state Precession) and FLASH (Fast Low Angle Shot) imaging sequences. To visualize fiber orientation in each heart, a diffusionweighted MRI sequence was also performed. Capitalizing on the combined advantages of both FISP (consistent volume boundaries) and FLASH sequences (high internal contrast), we produced geometric segmentations of cardiac tissue, blood, and transmural plunge needle geometries using the Seg3D open-source software package (https://www.sci.utah.edu/software/seg3d).

\subsection{Geometric Registration}

At the conclusion of each experiment, the locations of the linear torso surface electrode strips, preselected sock electrodes, and plunge needle insertion sites on the cardiac surface were digitally recorded using a Microscribe threedimensional digitizer (Solution Technologies, Oella, MD, USA). In addition, landmark sites including the location of the occlusion site, major epicardial coronary arteries, and the outline of the myocardial shape were also captured using the digitizer. Once the locations of the plunge needles were recorded, they were replaced with plastic spacers.

\subsection{Signal Processing and Data Visualiza- tion}

The electrical signals recorded during the study were processed in "Preprocessing Framework for Electrograms Intermittently Fiducialized from Experimental Recordings" (PFEIFER) program, an open-source MATLABbased signal processing platform designed to process bioelectric signals acquired from cardiac experiments. [6] Using PFEIFER, we were able to calibrate, baseline correct, filter, and mark specific time instances within cardiac signals for analysis.

Finally, the processed experimental signals were mapped to the identified electrode locations within the heart, on the epicardial surface of the heart, and on the torso surface. Full experimental model datasets were then visualized using map3d (sci.utah.edu/software/map3d) or SCIRun (sci.utah.edu/software/scirun) open-source software packages which provided for extensive spatial exploration of the results.

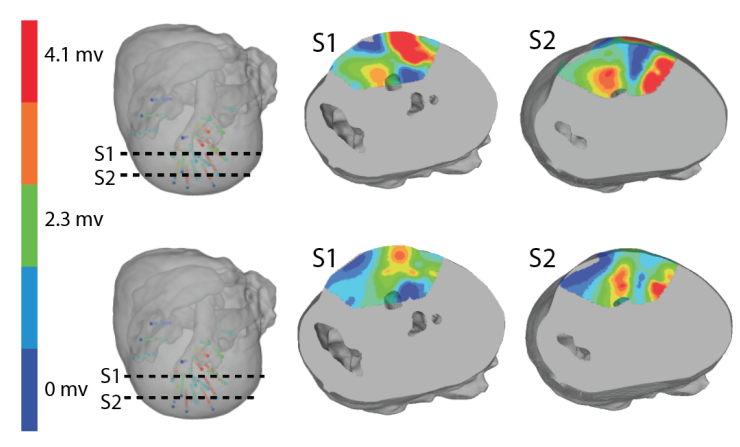

Figure 1. Regions of ischemia detected within the myocardium. ST\%40 values are indicated by the color, as shown in the scale bar. The dashed lines indicate the slice levels captured in the adjacent projections. Row 1: Bruce protocol. Row 2: Dobutamine Protocol. The superior edge of each slice corresponds to the anterior surface in the leftmost column.

\section{Results}

With this experimental preparation, we were able to simultaneously record from all three aforementioned regions with high resolution and adequate coverage. Ischemic control was achieved and four transient episodes of ischemia were induced for each animal.

Figure 1 shows one result to illustrate the differences in the region of ischemia created during the BRUCE and Dobutamine stress tests. During peak ischemic stress, the region identified as ischemic based on elevated ST\%40 potentials were larger following the dobutamine protocol than in the BRUCE protocol. Figure 2 shows how these features also propagated to the epicardial and body surfaces with the expected loss of localization.

\section{Discussion}

In this study, we proposed a novel experimental preparation to characterize and understand the electrical signals of myocardial ischemia within the heart and on the epicardial and body surfaces. In specific, we tested the hypothesis that different clinical cardiac stress tests cause different electrical signatures detectable on the body surface. Our results show that the BRUCE and dobutamine stress tests produce different amounts and spatial distributions of ischemia with comparable heart rates. The largest differences were visible within the myocardium, as shown in the number and locations of extrema in Figure 1. Epicardial and torso surface differences were also visible but limited to amplitudes of shared features. The dobutamine stress tests produced body surface signals with significant amounts of depression, while the BRUCE protocols produced only relatively mild depression. 


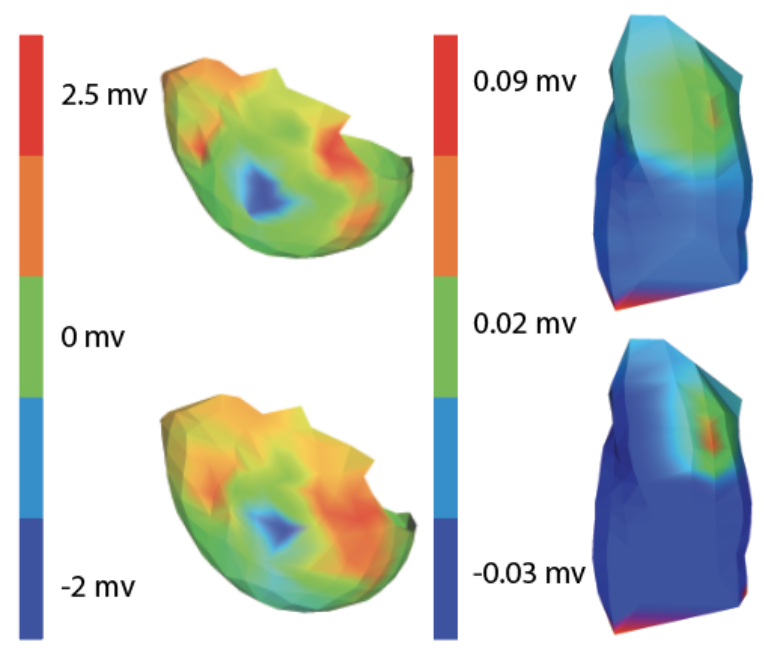

Figure 2. Regions of ischemia detected on the epicardial surface and the torso surface. Again, color indicates amplitude of the local ST\%40 values Row 1: Epicardial and Torso measurements from a Bruce Protocol. Row 2: Epicardial and Torso Measurements from a dobutamine protocol. Results are from the same cases as in Figure 1

These findings suggest different means of stressing the heart require unique diagnostic criteria to detect and monitor myocardial ischemia. This finding underscores a recurring theme that the process of ischemia detection and development is more complicated than conventional explanations have indicated. Our previous results have already demonstrated that non-transmural ischemia arises from multiple regions with the myocardium and shows complex spatio-temporal progression.[7]

Another important breakthrough in this study was the simultaneous recordings from within the heart, on the heart surface, and on the body surface during a controlled ischemic intervention. To date, we know of no published results documenting such high resolution with a similar protocol. The datasets used in this study will be ideal test datasets for other methods of detecting ischemia, including electrocardiographic imaging. The high resolution, ischemic control, and simultaneous recordings in multiple region make these datasets extremely valuable to the community at large.

This project was limited by the small number of experiments performed so far. This study was also limited in direct clinical translation because of the animal torso shape and other anatomical features. Future directions of this project will include more experiments performed with both stress protocols.

\section{Acknowledgements}

Support for this research comes from the NIH NIGMS Center for Integrative Biomedical Computing (www.sci.utah.edu/cibc), NIH NIGMS grant no. P41GM103545 and the Nora Eccles Treadwell Foundation for Cardiovascular Research.

\section{References}

[1] Roth GA, Huffman MD, Moran AE, Feigin V, Mensah GA, Naghavi M, Murray CJL. Global and regional patterns in cardiovascular mortality from 1990 to 2013. Circulation 2015; 132(17):1667-1678. ISSN 15244539.

[2] McCarthy BD, Wong JB, Selker HP. Detecting acute cardiac ischemia in the emergency department. Journal of General Internal Medicine jul 1990;5(4):365-373. ISSN 1525-1497.

[3] Akkerhuis K, Simoons M. Exercise electrocardiography and exercise testing. Comprehensive Electrocardiology 2011; $1: 1677-1719$.

[4] Okin PM, Ameisen O, Kligfield P. A modified treadmill exercise protocol for computer-assisted analysis of the ST segment/heart rate slope: Methods and reproducibility. Journal of Electrocardiology oct 1986;19(4):311-318. ISSN 00220736.

[5] Secknus MA, Marwick TH. Evolution of dobutamine echocardiography protocols and indications: Safety and side effects in 3,011 studies over 5 years. Journal of the American College of Cardiology 1997;29(6):1234-1240. ISSN 07351097.

[6] Rodenhauser A, Good WW, Zenger B, Tate J, Aras K, Burton B, Macleod RS. PFEIFER: Preprocessing framework for electrograms intermittently fiducialized from experimental recordings. Journal of Open Source Software 2018; $3(21): 472$.

[7] Aras K, Burton B, Swenson D, MacLeod R. Spatial organization of acute myocardial ischemia. Journal of Electrocardiology 2016;49(3):323-336. ISSN 15328430.

Address for correspondence:

Brian Zenger

72 Central Campus Dr, Salt Lake City, UT 84112

zenger@sci.utah.edu 